\title{
Artículos de ínvestigación
}

\section{Longitud cervical o puntaje de bishop en la predicción de parto pretérmino inminente en pacientes sintomáticas.}

\section{Cervical length or bishop score in prediction of imminent preterm delivery in symptomatic patients.}

\author{
Eduardo Reyna-Villasmil ${ }^{1}$, Jorly Mejia-Montilla ${ }^{2}$, Nadia Reyna-Villasmil ${ }^{2}$, Duly \\ Torres-Cepeda ${ }^{1}$, Martha Rondón-Tapia ${ }^{1}$, Danaisy Cabrera-Montes de Oca ${ }^{3}$, Isoled \\ Herrera Pineda 4', Carlos Briceño-Pérez ${ }^{2}$.
}

\footnotetext{
1 Servicio de Ginecología y Obstetricia. Hospital Central “Dr. Urquinaona”. Maracaibo. VENEZUELA.

${ }^{2}$ Facultad de Medicina. La Universidad del Zulia. Maracaibo. VENEZUELA.

${ }^{3}$ Facultad de Ciencias de la Salud. Universidad Técnica de Manabí. Portoviejo. ECUADOR.

${ }^{4}$ Facultad de Ciencias Sociales y de la Salud. Universidad Estatal Península de Santa Elena. La Libertad. ECUADOR.
}

\section{Correspondencia: \\ Dr. Eduardo Reyna-Villasmil. Hospital Central "Dr. Urquinaona".Final Av. El Milagro. Maracaibo, Estado Zulia, Venezuela. e-mail: sippenbauch@gmail.com}

\section{RESUMEN}

OBJETIVO: Comparar la longitud cervical con el puntaje de Bishop en la predicción de parto pretérmino inminente en pacientes sintomáticas.

MÉTODOS: Se seleccionaron mujeres con embarazos simples de 24 - 35 semanas, con amenaza de parto pretérmino y membranas integras. Antes del inicio de cualquier tratamiento, todas fueron sometidas a examen digital del cuello uterino y determinación ecográfica transvaginal de la longitud cervical. La principal variable de estudio fue la frecuencia de parto inminente (en los 7 días siguientes a la evaluación). Se evaluaron las características generales, puntaje de Bishop y valores de la longitud cervical

RESULTADOS: Fueron seleccionadas 481 mujeres, 119 participantes presentaron parto pretérmino inminente (grupo A) y 362 pacientes presentaron partos más allá de los 7 días (grupo B). El intervalo entre la evaluación y el parto fue de 4,3 +/- 1,6 días en el grupo A y 56,3 +/- 27,2 días en el grupo B ( $p<0,0001)$. Las pacientes del grupo A tenían valores más bajos de longitud cervical comparado con las pacientes del grupo $B$ ( $p<0,0001$ ). Este grupo también presentó valores más elevados de puntaje de Bishop $(p<0,0001)$. La longitud cervical tenía un área bajo la curva mayor para la predicción (0,972, intervalo de confianza 95\%, 0,772 - 1,000) comparado con el puntaje de Bisho (0,825, intervalo de confianza 95\%, 0,783-0,870; $p=0,0137)$.

CONCLUSIÓN: La longitud cervical es más útil en la predicción de parto pretérmino inminente en pacientes sintomáticas comparado con el puntaje de Bishop.

PALABRAS CLAVE: Longitud cervical; Puntaje de Bishop; Predicción; Parto pretérmino; amenaza de parto pretérmino.. 


\section{ABSTRACT}

OBJECTIVE: To compare the cervical length with Bishop score in prediction of imminent preterm delivery in symptomatic patients.

METHODS: Women with single pregnancies of 24-35 weeks were selected, with the diagnosis of threatened preterm labor and intact membranes. Before the start of any treatment, all were submitted to the cervical digital examination and transvaginal ultrasound determination of cervical length. The main variable of the study was the frequency of imminent delivery (in the 7 days following evaluation). General characteristics, Bishop score, and cervical length values were evaluated.

RESULTS: A total of 481 women were selected, 119 patients presented imminent delivery (group A) and 362 patients delivered after 7 days (group B). the interval between evaluation and delivery was $4.3+/-1.6$ days in group $A$ and $56.3+/-27.2$ days in group $B(p<0.0001)$. Patients in group $A$ had lower values of cervical length compared with patients in group B $(p<0.0001)$. This group also presented higher values of Bishop score ( $p$ $<0.0001)$. Cervical length had a mayor area under for prediction $(0.972,95 \%$ confidence interval, $0.772-1.000)$ compared with Bishop score $(0.825,95 \%$ confidence interval, $0.783-0.870, p=0.0137)$.

CONCLUSION: Cervical length is more useful in the prediction of imminent preterm delivery in symptomatic patients compared to the Bishop score.n.

KEY WORDS: Cervical length; Bishop's score; prediction; pre-term delivery; Threatened preterm labor.

\section{INTRODUCCIÓN}

La amenaza de parto pretérmino es un diagnóstico común que lleva a hospitalización durante el embarazo. Aunque más de la mitad de las pacientes con esta condición llegaran al final del embarazo, aproximadamente $20 \%$ tendrán partos antes del término del embarazo, a pesar del uso de medidas farmacológicas y no farmacológicas para evitarlo ${ }^{1}$. Por lo tanto, tener una prueba con la capacidad de predecir la posibilidad del parto pretérmino es importante porque intervenciones, como tocólisis, administración de esteroides para inducir la maduración pulmonar fetal y transferencia a centros especializados, pueden mejorar los resultados perinatales ${ }^{2}$.

El examen digital del cuello uterino, que evalúa los componentes del puntaje de Bishop (PB), es el método tradicional para evaluar los cambios cervicales que llevan al diagnóstico de parto pretérmino. Pero esta evaluación clínica tiene poca sensibilidad y especificidad para predecir el parto pretérmino ${ }^{3}$. A fines de la década de 1980, fue introducida la exploración ecográfica cervical como una técnica diagnostica prometedora. Estudios observacionales afirman que es superior al examen digital en la predicción de parto pretérmino, ya que la medición de la longitud cervical (LC) es más precisa, reproducible y presenta menos variaciones intraobservador comparado con el PB 4-6. Todo esto a pesar de que el examen digital permite evaluar la consistencia y posición cervical y la presión que procede del feto sobre el cuello uterino.

Aunque existe evidencia que, en pacientes con embarazos simples, la LC no es mejor predictor del parto pretérmino que el examen digital y se puede recomendar su uso rutinario, especialmente en vista de su baja sensibilidad ${ }^{7}$. Sin embargo, existen datos limitados sobre la capacidad de predicción de la LC o el examen digital del parto pretérmino en los siguientes 7 días en pacientes con contracciones uterinas y amenaza de parto pretérmino. Por lo tanto, el objetivo de la investigación fue comparar la longitud cervical con el puntaje de Bishop en la predicción de parto pretérmino inminente en pacientes sintomáticas.

\section{MÉTODOS}

Este fue un estudio de cohortes prospectivo realizado en el Hospital Central "Dr. Urquinaona", Maracaibo, Venezuela, de junio de 2014 a marzo de 2018. Para esto fueron seleccionadas y evaluadas en forma consecutiva mujeres con embarazos simples entre 24 y 35 semanas, determinado por ecografía del comienzo del segundo trimestre, con diagnóstico clínico de amenaza de parto pretérmino, con hasta 3 contracciones uterinas en 30 minutos, dilatación cervical menor de 3 centímetros por examen físico, borramiento menor del $80 \%$ y membranas integras. $\mathrm{El}$ estudio fue aprobado por la junta de revisión institucional de la Facultad de Medicina de La Universidad del Zulia y el Comité de Ética del hospital. 
El consentimiento informado por escrito fue obtenido al momento de la selección de cada participante para el estudio.

Los criterios de exclusión fueron: mujeres con embarazos múltiples, antecedentes de uso de progesterona o cualquier otro tocolítico en el embarazo actual, insuficiencia cervical conocida o antecedente de cerclaje, rotura prematura de membranas, anomalías uterinas, placentación anormal, alteraciones cardiacas maternas, enfermedad inflamatoria o infecciosa activa, hipertensión y diabetes pre gestacional o gestacional, restricción del crecimiento intrauterino del feto, anomalías fetales congénitas, alteraciones del volumen de líquido amniótico, corioamnionitis aguda y edad gestacional desconocida. También fueron excluidas del estudio aquellas pacientes que se negaron a participar.

En la hora siguiente a la admisión hospitalaria, y antes del inicio de cualquier tratamiento, las participantes fueron sometidas a examen del cuello uterino con espéculo estéril, realizándose las pruebas de helecho y papel de nitracina para descartar rotura de membranas. Inmediatamente después se practicó el examen digital, el cual fue realizado por el personal de guardia y confirmado por uno de tres investigadores de forma inmediata luego de esta evaluación, para evaluar dilatación, maduración, consistencia y posición del cuello uterino, además del encajamiento fetal para calcular el PB. En la hora siguiente al ingreso, fueron sometidas a evaluación ecográfica fetal transabdominal para determinar presentación fetal, sitio de inserción placentario, medición de circunferencia cefálica y abdominal, longitud del fémur e índice de líquido amniótico. Luego de esto, se inició monitoreadas la frecuencia cardiaca fetal continuo y evaluación de las características de las contracciones uterinas por tocodinamometría externa.

La evaluación de la LC por ecografía transvaginal fue hecha inmediatamente después del examen digital. Las embarazadas fueron colocadas en posición de litotomía dorsal con la vejiga vacía con equipo de ecografía Voluson® V730 Expert (GE Healthcare, EE. UU.). La evaluación fue realizada colocando el transductor transvaginal de 7,5 $\mathrm{MHz}$ en el fórnix vaginal anterior, retirándolo lentamente para obtener una vista sagital del cuello uterino. En ausencia de contracciones uterinas, las mediciones fueron realizadas orientando el transductor para ver el orificio cervical interno y externo en el mismo plano. La imagen fue ampliada a $75 \%$ de la pantalla y la LC fue medida como una línea recta entre dos marcadores electrónicos con los puntos más alejados e identificada por la sonolucencia del canal cervical. Se obtuvieron tres imágenes y la que mostraba la longitud más corta fue la utilizada. El funneling cervical fue descrito cuando el borde lateral del embudo cervical tenía al menos 3 milímetros de longitud 7 . Todas las mediciones fueron realizadas por un solo especialista en medicina materno-fetal perteneciente a la investigación y que no participo en la atención clínica de las participantes. Los miembros del equipo hospitalario que realizaban la atención desconocían los resultados de la LC, pero conocían el PB. Por lo tanto, las mediciones ecográficas no influyeron en el manejo de las participantes.

Inicialmente, todas las participantes de la investigación fueron ingresadas para ser tratadas con reposo en cama en decúbito lateral izquierdo e hidratadas con $500 \mathrm{~mL}$ de solución de Ringer lactato. Si se documentaban cambios cervicales progresivos o las contracciones persistían por al menos 2 horas después del tratamiento, se hospitalizaban e iniciaba tratamiento tocolítico, con bloqueadores de los canales de calcio como terapia de primera línea. No se utilizó tratamiento tocolítico combinado. Durante la hospitalización se administraron dos dosis de $12 \mathrm{mg}$ de betametasona intramuscular con intervalo de 24 horas para inducir maduración pulmonar fetal. Los tocolíticos se suspendieron 48 horas después de la primera dosis de esteroides. No se utilizaron tocolíticos ni esteroides en embarazos mayores de 34 semanas.

Se elaboró un formulario que incluía estos datos: número de identificación, edad materna, edad gestacional al momento del examen, paridad, presencia de sangrado, antecedentes de parto pretérmino, examen cervical digital con determinación de los componentes del PB (dilatación y borramiento, estación y consistencia y posición cervical) y valores de la LC y presencia de funneling. La principal variable de estudio fue la frecuencia de parto inminente (aquel que se produjo en los 7 días siguientes a la evaluación). También fueron incluidos datos sobre el peso de los recién nacidos al nacer e intervalo de tiempo entre la evaluación y el parto. Todos los datos se registraron y almacenaron para el análisis posterior y no estaban disponibles para el personal que atendía las pacientes durante la hospitalización.

Las variables categóricas se analizaron usando las pruebas chi-cuadrado o prueba $U$ de MannWhitney. Las variables continuas con distribución normal fueron comparadas con la prueba t de Student. 
Aquellas variables con distribución diferente a la normal fueron comparadas con la prueba suma de rangos de Wilcoxon. Se utilizaron las curvas operadorreceptor para determinar el mejor punto de corte y evaluar la precisión diagnóstica de LC y el PB en la predicción del parto prematuro espontáneo en los siguientes 7 días de la evaluación. La prueba de Hanley McNei fue utilizada para comparar las diferencias en las áreas bajo la curva de ambas pruebas. También fueron calculadas la sensibilidad, especificidad, valores predictivos positivos $y$ negativos junto a los intervalos de confianza del 95\%. Se realizó el análisis de regresión lineal para evaluar como las dos variables estudiadas modificaban el intervalo de tiempo entre la evaluación y el parto, para lo que se realizaron análisis preliminares para garantizar que no se violaban los supuestos obligatorios. El nivel alfa de significancia estadística fue de $p<0,05$. Todos los análisis fueron realizados con el paquete estadístico SPSS $\AA$ versión 22.0 (SPSS Inc., EE. UU.).

\section{RESULTADOS}

Para el estudio se seleccionaron 518 pacientes de forma consecutiva, de las cuales 21 pacientes fueron excluidas por presentar rotura prematura de membranas, 5 pacientes con preeclampsia y una paciente con anomalías uterinas diagnosticadas durante el seguimiento. Además, otras 10 pacientes fueron excluidas por carecer datos completos de evaluación y/o seguimiento. Por lo tanto, para el análisis final fueron seleccionadas 481 mujeres, de las cuales 119 participantes (24,8\%) presentaron parto pretérmino en los 7 días siguientes a la evaluación (grupo A) y 362 pacientes (75,2\%) presentaron partos más allá de los 7 días (grupo B).

Las características de ambos grupos se muestran en la tabla 1. La frecuencia de antecedentes de parto pretérmino $(p=0,0186)$ y sangrado genital $(p=$ $0,0016)$ fueron significativamente mayores en las pacientes del grupo A comparado con aquellas del grupo $B$. El intervalo entre la evaluación y el parto fue de 4,3 +/- 1,6 días en el grupo A y 56,3 +/- 27,2 días en el grupo $B(p<0,0001)$. No se encontraron diferencias significativas en la edad materna, frecuencia de nuliparidad, hábito tabáquico antes del embarazo, índice de masa corporal, edad gestacional al momento de la evaluación y antecedente de uso de corticosteroides $(p=n s)$. El peso de los recién nacidos fue significativamente mayor en el grupo $B$ comparado con el grupo $A(p<0,0001)$.
Las mediciones de LC, presencia de funneling y variables asociadas al PB (tabla 2) demostraron que las pacientes del grupo $A$ tenían valores más bajos de LC comparado con las pacientes del grupo B (figura $1 ; p<0,0001)$. La frecuencia de funneling cervical también fue mayor en las pacientes del grupo $A(p<$ 0,0001). Por otro lado, este grupo de pacientes presentó valores significativamente más elevados de dilatación cervical, borramiento cervical y PB (figura 2; $p<0,0001)$.

En la figura 1 se muestra la curva operadorrespuesta de la $L C$ y el PB para la predicción de parto pretérmino inminente. Se observo que la LC tenía un área bajo la curva significativamente mayor $(0,972$, intervalo de confianza del 95\%, 0,772 - 1,000) comparado con el PB $(0,825$, intervalo de confianza, $0,783-0,870)$. La comparación de la capacidad de discriminación de estas pruebas demostró que había diferencias significativas en las áreas bajo la curva ( $p$ $=0,0001$ ). Los resultados también demuestran que un valor de corte para la LC de 20 milímetros presentaba mayor especificidad, valor predictivo positivo y negativo, razón de probabilidad y eficacia diagnostica que el PB de 4 puntos (tabla 3).

Finalmente, el análisis de regresión lineal utilizado para determinar cuál de las variables (PB y LC) que estaban asociadas que permiten para predecir el intervalo de tiempo entre la evaluación y el parto, demostró que el modelo de predicción fue significativo para la LC $(X=-15.290+(1.940$ * LC $))$, que expresaba $27,6 \%$ de la variación del intervalo ( $\mathrm{r} 2=0,277, \mathrm{r} 2$ ajustado $=0,276)$. Mientras que el PB $(x=74.661$ $(9.966$ * PB)) mostró un efecto sobre la variación del intervalo menor ( $r 2=0,124, \mathrm{r} 2$ ajustado $=0,122$ ).

\section{DISCUSIÓN}

Los resultados de la investigación sugieren que la LC es superior que el PB para la predicción del parto pretérmino inminente, lo que suministra elementos para considerarlo una herramienta útil en la predicción del parto pretérmino en los siguientes 7 días.

Los métodos convencionales para predecir el parto pretérmino dependen de la historia obstétrica, factores demográficos, evaluación de las contracciones uterinas y examen digital del cuello uterino, los cuales son poco sensibles e inespecíficos (8). Aunque el examen digital, el cual es subjetivo y necesita de experiencia, es parte de la evaluación rutinaria de las pacientes con amenaza de parto pretérmino, en muchas ocasiones conduce a predicción imprecisa del parto inminente ${ }^{4,9}$. La 
evaluación ecográfica del cuello uterino es un método objetivo y no invasivo que evalúa los cambios en la morfología del cuello uterino ${ }^{10}$. La LC es la característica más común estudiada en la predicción del parto pretérmino. Esta técnica incluye identificación del orificio cervical interno y externo además del canal cervical con la medición de la distancia entre ambos orificios en milímetros ${ }^{11,12}$. Existen diferentes valores de corte de LC y la evidencia sugiere que es una herramienta objetiva en la predicción del parto pretérmino 6,13,14.

Hasta la fecha, la literatura que compara la LC por ecografía transvaginal con el examen digital en embarazadas con amenaza de parto pretérmino inminente es escasa y contradictoria. La mayoría de las investigaciones están centrados en la predicción de parto a término espontaneo ${ }^{15,16}$. Dos estudios demostraron que un valor de corte de 25 milímetros para la LC tenía sensibilidad superior al $80 \%$ para partos pretérminos que ocurrieron en las 48 horas siguientes con especificidad de alrededor del $70 \%$ 17,18. Existe evidencia que el riesgo de parto pretérmino está relacionado con la LC por lo que puede ser un indicador útil y rentable para identificar el riesgo de parto antes del término del embarazo ${ }^{19}$. Una revisión sistemática demostró que cuanto más corta es la LC, mayor es el riesgo de parto pretérmino y un valor de corte de menor de 25 milímetros es el de mayor utilidad ${ }^{20}$. Este valor de corte también se ha utilizado en otros estudios ${ }^{21}$. Sin embargo, la determinación de un cuello uterino corto en el tercer trimestre también está asociado a parto pretérmino ${ }^{22}$. El valor de corte de 20 milímetros mostró una especificidad superior. Es importante resaltar que estos estudios incluyen aspectos que han demostrado considerable heterogeneidad, a pesar de la similitud en la metodología de evaluación de la LC y las diferencias en los criterios de selección. No obstante, los valores de la razón de posibilidad positiva y negativa en esta investigación fueron marcadamente superiores a lo reportado previamente ${ }^{13}$. Por otra parte, algunas investigaciones han propuesto que la LC es un mejor predictor de parto pretérmino en intervalos de tiempo más largo que en periodos más cortos ${ }^{7}$.

El examen manual y el PB pueden tener variaciones inter-operador generalmente mayores que la ecografía 4,7. La comparación de la reproducibilidad de la ecografía y el examen cervical digital encontró que dos examinadores concordaron dentro de 1 milímetro en $74 \%$ de los exámenes ecográficos en comparación con $35 \%$ de los exámenes digitales ${ }^{18}$. Si bien se afirma que el examen manual es útil para predecir el parto pretérmino ${ }^{23-25}$, su alta variabilidad limita su utilidad potencial 4,5 . No obstante, varios estudios han demostrado que tienen valores predictivos similares a la LC. Una de ellos evaluó la capacidad de predicción de la LC y el PB en pacientes con amenaza de parto pretérmino, contracciones uterinas y membranas integras, encontrado que ambas tenían áreas bajo la curva $(p=0,028)$ similares para la predicción del parto antes de las 37 semanas, pero en las nulíparas la diferencia si fue significativa $(p=0,04)^{26}$. Un metaanálisis demostró que el valor predictivo del examen digital y la LC en pacientes de bajo riesgo y sintomáticas antes del tratamiento concluyo la ecografía transvaginal que la mejora en la predicción en mujeres sintomáticas es insuficiente y sugieren que ambos métodos deben usarse en forma complementaria para disminuir la tasa de falsos negativos ${ }^{27}$. Finalmente, otro estudio q concluyó que un PB mayor o igual a 5 puntos tenía una capacidad de predicción de parto pretérmino a los 7 días similar la LC menor de 25 milímetros 28.

Dado que el método ecográfico es capaz de detectar pequeñas variaciones del cuello uterino comparado con cualquier parámetro clínico, esto permite clasificar a las pacientes con contracciones uterinas antes del término y membranas integras que tienen alto riesgo para un parto pretérmino inminente. No obstante, el PB sigue siendo una herramienta útil, en especial en aquellos centros donde no se dispone del personal o los equipos para la evaluación ecográfica del cuello uterino. En esta investigación el PB mostro una capacidad discriminatoria alta con valores superiores a los reportados previamente ${ }^{29}$.

Aunque ningún ensayo clínico aleatorizado ha demostrado que la medición de la LC reduzca la morbi-mortalidad perinatal, los hallazgos de esta investigación tienen importancia clínica, ya que pueden ser útiles para el manejo de las pacientes (uso de corticosteroides para la maduración pulmonar fetal y el inicio de tratamiento tocolítico inmediato) e identificar un grupo de mujeres de alto riesgo para futuros ensayos de intervenciones para reducir los efectos de la prematuridad.

El estudio tiene varias fortalezas. Todas las participantes fueron seguidas hasta el parto. Además, la evaluación clínica fue realizada en forma consecutiva y los médicos tratantes desconocían los resultados de la evaluación ecográfica del cuello uterino, lo cual limitó los posibles sesgos. Por otra parte, un número significativo de pacientes 
seleccionadas presentó parto pretérmino inminente por lo que también puede disminuir la posibilidad de errores en el análisis estadístico. Sin embargo, el estudio también presenta algunas debilidades que incluyen la ausencia de análisis de la variabilidad inter-observador en la medición de la LC. Debido a que el objetivo del estudio era comparar la efectividad pronostica de ambas pruebas en el parto pretérmino inminente en pacientes sintomáticas, se decidió limitar este aspecto. Además, este estudio fue realizado en un solo centro con población bastante homogénea, lo cual puede dificultar la generalización de los resultados. Tampoco fueron considerados otras características ecográficas cervicales ni los cambios de la LC, variables que también pueden ser útiles para predecir el parto pretérmino inminente.

\section{CONCLUSIÓN}

Los hallazgos de la investigación proporcionan evidencia que la longitud cervical es más útil en la predicción de parto pretérmino inminente en pacientes sintomáticas comparado con el puntaje de Bishop. Estos datos pueden ser la base para un tratamiento más intensivo en las pacientes que presentan mayor riesgo de parto antes de los 7 días. No obstante, son necesarias investigaciones complementarias para confirmar estos resultados.

\section{REFERENCIAS}

1. Howland RE, Angley M, Won SH, Wilcox W, Searing $\mathrm{H}$, Tsao TY. Estimating the hospital delivery costs associated with severe maternal morbidity in New York City, 2008-2012. Obstet Gynecol. 2018; 131(2): 242-252.

2. Patel SS, Ludmir J. Drugs for the treatment and prevention of preterm labor. Clin Perinatol. 2019; 46(2): 159-172.

3. How HY, Khoury JC, Sibai BM. Cervical dilatation on presentation for preterm labor and subsequent preterm birth. Am J Perinatol. 2009; 26(1): 1-6.

4. Holcomb WL Jr, Smeltzer JS. Cervical effacement: variation in belief among clinicians. Obstet Gynecol. 1991; 78(1): 43-5.

5. Stein W, Hellmeyer L, Schmidt S, Tekesin I. Intraobserver and interobserver reliability of transvaginal cervical length measurements and quantitative ultrasound tissue characterization of the cervix in the second and third trimester of pregnancy. Ultraschall Med. 2011; 32 Suppl 2: E169-74.
6. Gomez R, Galasso M, Romero R, Mazor M, Sorokin $\mathrm{Y}$, Gonçalves L, et al. Ultrasonographic examination of the uterine cervix is better than cervical digital examination as a predictor of the likelihood of premature delivery in patients with preterm labor and intact membranes. Am J Obstet Gynecol. 1994; 171(4): 956-64.

7. Crosby DA, Miletin J, Semberova J, Daly S. Is routine transvaginal cervical length measurement cost-effective in a population where the risk of spontaneous preterm birth is low? Acta Obstet Gynecol Scand. 2016; 95(12): 1391-1395.

8. McCabe ERB. Preventable preterm birth: A patient safety problem. Pediatrics. 2019; 143(5). pii: e20180940.

9. Rozenberg P, Gillet A, Ville Y. Transvaginal sonographic examination of the cervix in asymptomatic pregnant women: review of the literature. Ultrasound Obstet Gynecol. 2002; 19(3): 302-11.

10. Wax JR, Cartin A, Pinette MG. Biophysical and biochemical screening for the risk of preterm labor: An update. Clin Lab Med. 2016; 36(2): 369-83.

11. Ville $Y$, Rozenberg P. Predictors of preterm birth. Best Pract Res Clin Obstet Gynaecol. 2018; 52: 23-32.

12. Fuchs IB, Henrich W, Osthues K, Dudenhausen JW. Sonographic cervical length in singleton pregnancies with intact membranes presenting with threatened preterm labor. Ultrasound Obstet Gynecol. 2004; 24(5): 554-7.

13. Sotiriadis A, Papatheodorou S, Kavvadias A, Makrydimas $G$. Transvaginal cervical length measurement for prediction of preterm birth in women with threatened preterm labor: a metaanalysis. Ultrasound Obstet Gynecol. 2010; 35(1): 54-64.

14. Oskovi Kaplan ZA, Ozgu-Erdinc AS. Prediction of preterm birth: Maternal characteristics, ultrasound markers, and biomarkers: An updated overview. J Pregnancy. 2018; 2018: 8367571.

15. Rosati P, Ciliberti P, Buongiorno S, Alessio A, Mappa I, Guariglia L, et al. Ultrasonographic and clinical methods in the management of prolonged pregnancy. Minerva Ginecol. 2014; 66(2): 193-9.

16. Mukherji J, Bhadra A, Ghosh SK, Hazra A, Anant $M$, Bhattacharya $S K$, et al. Cervical length measurement in nulliparous women at term by ultrasound \& its relationship to spontaneous onset of labour. Indian J Med Res. 2017; 146(4): 498504. 
17. Boudhraa K, Rahouej H, Gara MF. Transvaginal ultrasound of the cervix in the estimation of severity of premature labour. Tunis Med. 2008; 86(8): 745-8.

18. Schmitz T, Kayem G, Maillard F, Lebret MT, Cabrol D, Goffinet F. Selective use of sonographic cervical length measurement for predicting imminent preterm delivery in women with preterm labor and intact membranes. Ultrasound Obstet Gynecol. 2008; 31(4): 421-6.

19. Werner EF, Hamel MS, Orzechowski K, Berghella V, Thung SF. Cost-effectiveness of transvaginal ultrasound cervical length screening in singletons without a prior preterm birth: an update. Am J Obstet Gynecol. 2015; 213(4): 554.e1-6.

20. Crane JM, Hutchens D. Transvaginal sonographic measurement of cervical length to predict preterm birth in asymptomatic women at increased risk: a systematic review. Ultrasound Obstet Gynecol. 2008; 31(5): 579-87.

21. Enakpene CA, DiGiovanni L, Jones TN, Marshalla M, Mastrogiannis D, Della Torre M. Cervical cerclage for singleton pregnant patients on vaginal progesterone with progressive cervical shortening. Am J Obstet Gynecol. 2018; 219(4): 397.e1397.e10.

22. Benshalom-Tirosh N, Tirosh D, Aricha-Tamir B, Weintraub AY, Erez O, Mazor M, et al. The clinical utility of sonographic cervical length in the management of preterm parturition at 28-32 weeks of gestation. J Matern Fetal Neonatal Med. 2015; 28(16): 1929-33.

23. Blondel B, Le Coutour X, Kaminski M, Chavigny C, Bréart G, Sureau C. Prediction of preterm delivery: is it substantially improved by routine vaginal examinations? Am J Obstet Gynecol. 1990; 162(4): 1042-8.

24. Hartmann K, Thorp JM Jr, McDonald TL, Savitz DA, Granados JL. Cervical dimensions and risk of preterm birth: a prospective cohort study. Obstet Gynecol. 1999; 93(4): 504-9.

25. Raba G, Kotarski J. Evaluation of risk factors can help to predict preterm delivery within 7 days in women hospitalized for threatened preterm labour. J Matern Fetal Neonatal Med. 2016; 29(19): 31426.

26. Sharvit M, Weiss R, Ganor Paz Y, Tzadikevitch Geffen K, Danielli Miller N, Biron-Shental T. Vaginal examination vs. cervical length - which is superior in predicting preterm birth? J Perinat Med. 2017; 45(8): 977-983.

27. Reiter E, Nielsen KA, Fedder J. Digital examination and transvaginal scan - competing or complementary for predicting preterm birth? Acta Obstet Gynecol Scand. 2012; 91(4): 428-38.

28. Adhikari K, Bagga R, Suri V, Takhtani M. Cervical length compared to Bishop's score for prediction of pre-term birth in women with pre-term labour. J Obstet Gynaecol. 2011; 31(3): 213-6.

29. Lotfi G, Faraz S, Nasir R, Somini S, Abdeldayem $R M$, Koratkar $R$, et al. Comparison of the effectiveness of a PAMG-1 test and standard clinical assessment in the prediction of preterm birth and reduction of unnecessary hospital admissions. J Matern Fetal Neonatal Med. 2019; 32(5): 793-797. 
TABLAS

TABLA 1.

CARACTERÍSTICAS GENERALES DE LAS PACIENTES

EN ESTUDIO.

\begin{tabular}{|c|c|c|c|}
\hline $\begin{array}{l}\text { Promedio +/- } \\
\text { desviacion estandar }\end{array}$ & $\begin{array}{l}\text { GRUPO A. } \\
\text { Parto antes } \\
\text { de } 7 \text { días } \\
(\mathrm{n}=113) \\
\end{array}$ & $\begin{array}{l}\text { GRUPO B. } \\
\text { Parto después } \\
\text { de } 7 \text { días. } \\
(\mathrm{n}=362)\end{array}$ & $p$ \\
\hline Edad materna, anos & $28,5+/-5,7$ & $29,4+/-7,1$ & 0,2196 \\
\hline Nuliparidad, n (\%) & $62(54,8)$ & $213(58,8)$ & 0,8274 \\
\hline $\begin{array}{l}\text { Antecedente de parto } \\
\text { pretérmino, } \mathrm{n}(\%)\end{array}$ & $27(23,9)$ & $50(13,8)$ & 0,0186 \\
\hline $\begin{array}{l}\text { Habito tabáquico antes } \\
\text { del embarazo, } \mathrm{n}(\%)\end{array}$ & $19(16,8)$ & $54(14,9)$ & 0,6545 \\
\hline Indice de masa corporal, $\mathrm{Kg} / \mathrm{m} 2$ & $27,4+/-6,4$ & $28,4+/-5,0$ & 0,0843 \\
\hline $\begin{array}{l}\text { Edad gestacional al } \\
\text { momento de la evaluación, semanas }\end{array}$ & $29,1+/-2,7$ & $29,5+/-3,1$ & 0,2181 \\
\hline $\begin{array}{l}\text { Intervalo entre la evaluación y } \\
\text { el parto, días }\end{array}$ & $4,3+/-1,6$ & $56,3+/-27,7$ & 0,0001 \\
\hline Sangrado genital, n (\%) & $65(57,5)$ & $146(40,3)$ & 0,0016 \\
\hline Uso de corticosteroides, n (\%) & $67(59,3)$ & $195(53,1)$ & 0,3309 \\
\hline $\begin{array}{l}\text { Peso del recién nacido } \\
\text { al nacer, gramos }\end{array}$ & $1900+/-844$ & $2627+/-773$ & 0,0001 \\
\hline
\end{tabular}

TABLA 2.

VARIABLES OBSTÉTRICAS EN CADA UNO DE LOS GRUPOS.

\begin{tabular}{llll}
\hline \hline $\begin{array}{l}\text { Promedio +/- } \\
\text { desviacion estandar }\end{array}$ & $\begin{array}{l}\text { GRUPO A. } \\
\text { Parto antes } \\
\text { de 7 días } \\
(\mathrm{n}=113)\end{array}$ & $\begin{array}{l}\text { GRUPO B. } \\
\text { Parto después } \\
\text { de 7 días. } \\
(\mathrm{n}=362)\end{array}$ & $\mathrm{p}$ \\
\hline Longitud cervical, milímetros & $18,4+/-5,9$ & $34,3+/-5,7$ & $<0,0001$ \\
Funneling cervical, $\mathrm{n}(\%)$ & $61(53,9)$ & $68(18,7)$ & $<0,0001$ \\
\hline Dilatación cervical, centímetros & $1,8+/-0,5$ & $0,8+/-0,4$ & $<0,0001$ \\
Borramiento cervical, porcentaje & $42,6+/-18,4$ & $12,2+/-7,7$ & $<0,0001$ \\
Puntaje de Bishop, puntos & $4,2+/-1,1$ & $2,8+/-0,9$ & $<0,0001$ \\
\hline \hline
\end{tabular}


TABLA 3.

PRECISIÓN PRONOSTICA DE PARTO ANTES DE LOS 7 DÍAS DE LA LONGITUD CERVICAL Y LA PRUEBA DE BISHOP EN PACIENTES CON AMENAZA DE PARTO PRETÉRMINO.

\begin{tabular}{|c|c|c|c|c|c|c|c|c|c|}
\hline $\begin{array}{l}\text { \% } \\
\text { (IC95\%) }\end{array}$ & $\begin{array}{l}\text { Área bajo } \\
\text { la curva }\end{array}$ & $\begin{array}{l}\text { Valor de } \\
\text { corte }\end{array}$ & Sensibilidad & Especificidad & $\begin{array}{l}\text { Valor } \\
\text { predictivo } \\
\text { positivo }\end{array}$ & $\begin{array}{l}\text { Valor } \\
\text { predictivo } \\
\text { negativo }\end{array}$ & $\begin{array}{l}\text { Razón de } \\
\text { probabilidad } \\
\text { positiva }\end{array}$ & $\begin{array}{l}\text { Razón de } \\
\text { probabilida } \\
\text { d } \\
\text { negativa }\end{array}$ & $\begin{array}{l}\text { Efi } \\
\text { prc }\end{array}$ \\
\hline $\begin{array}{l}\text { Longitud } \\
\text { cervical }\end{array}$ & $\begin{array}{l}0,972 \\
0,772-1,000\end{array}$ & $\begin{array}{l}20 \\
\text { milímetros }\end{array}$ & $\begin{array}{l}63,2 \\
54,0-71,4\end{array}$ & $\begin{array}{l}99,7 \\
98,5-100,0\end{array}$ & $\begin{array}{l}98,6 \\
92,6-99,8\end{array}$ & $\begin{array}{l}89,6 \\
86,2-92,2\end{array}$ & $\begin{array}{l}226,8 \\
32,1-1626,8\end{array}$ & $\begin{array}{l}0,37 \\
0,31-0,45\end{array}$ & $\begin{array}{l}91 \\
88\end{array}$ \\
\hline $\begin{array}{l}\text { Puntaje } \\
\text { de Bishop }\end{array}$ & $\begin{array}{l}0,825 \\
0,783-0,870\end{array}$ & 4 puntos & $\begin{array}{l}70,8 \\
61,8-78,4\end{array}$ & $\begin{array}{l}75,7 \\
71,0-79,8\end{array}$ & $\begin{array}{l}47,6 \\
40,2-55,1\end{array}$ & $\begin{array}{l}89,3 \\
85,3-92,2\end{array}$ & $\begin{array}{l}2,91 \\
2,34-3,62\end{array}$ & $\begin{array}{l}0,39 \\
0,29-0,52\end{array}$ & $\begin{array}{l}74 \\
70\end{array}$ \\
\hline
\end{tabular}

FIGURA 1. Valores de longitud cervical en cada uno de los grupos.

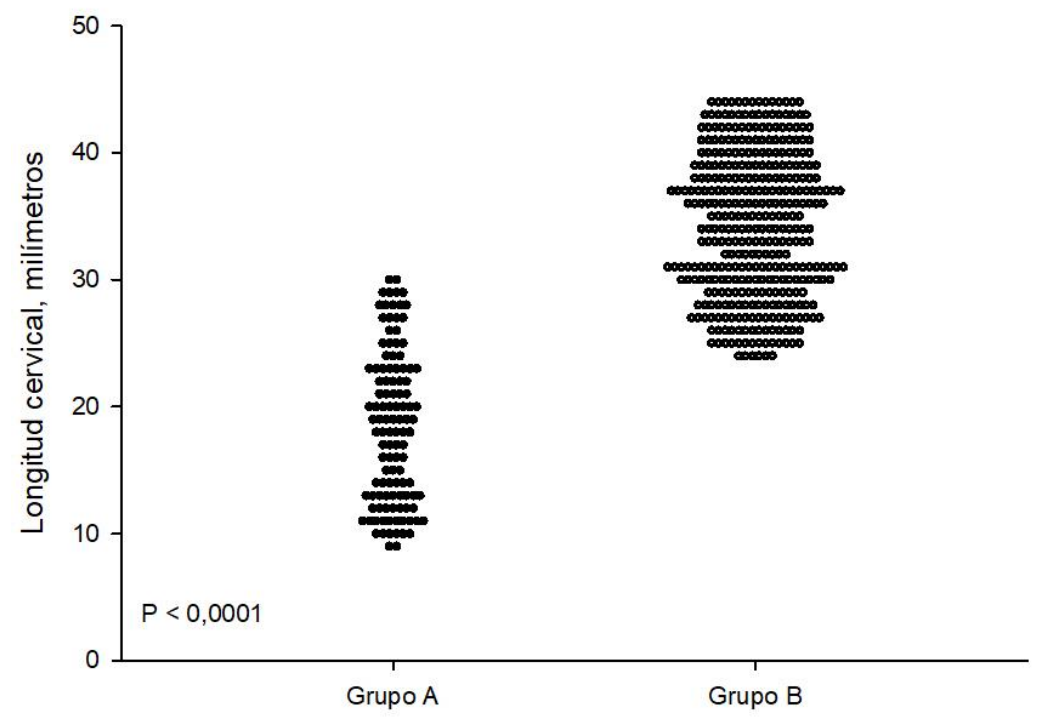


FIGURA 2. Valores de puntaje de Bishop en cada uno de los grupos.

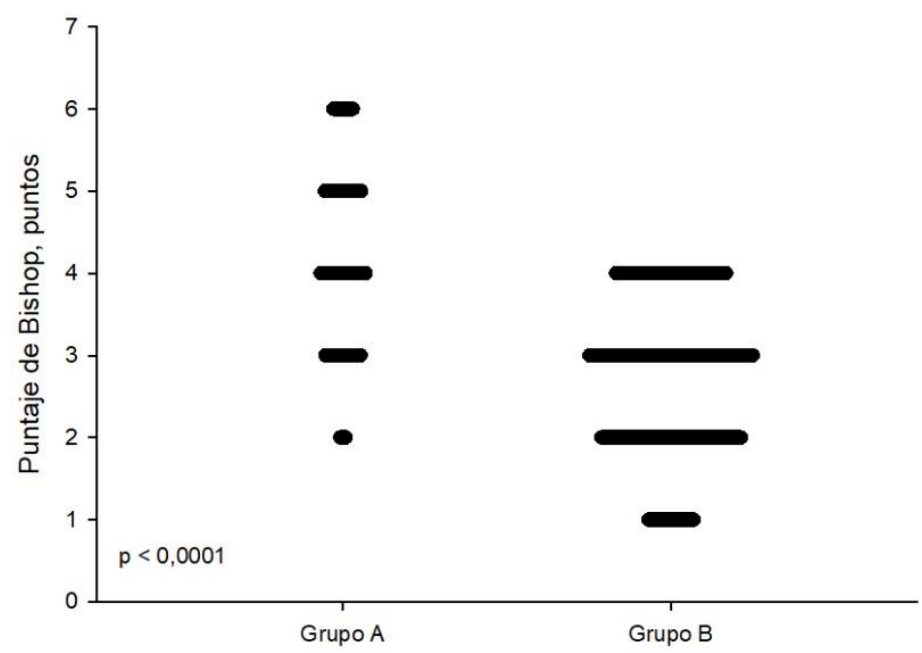

FIGURA 3. Curva operador-respuesta de la capacidad pronostica de la longitud cervical y el puntaje de Bishop en la predicción de parto pretérmino inminente en pacientes sintomáticas.

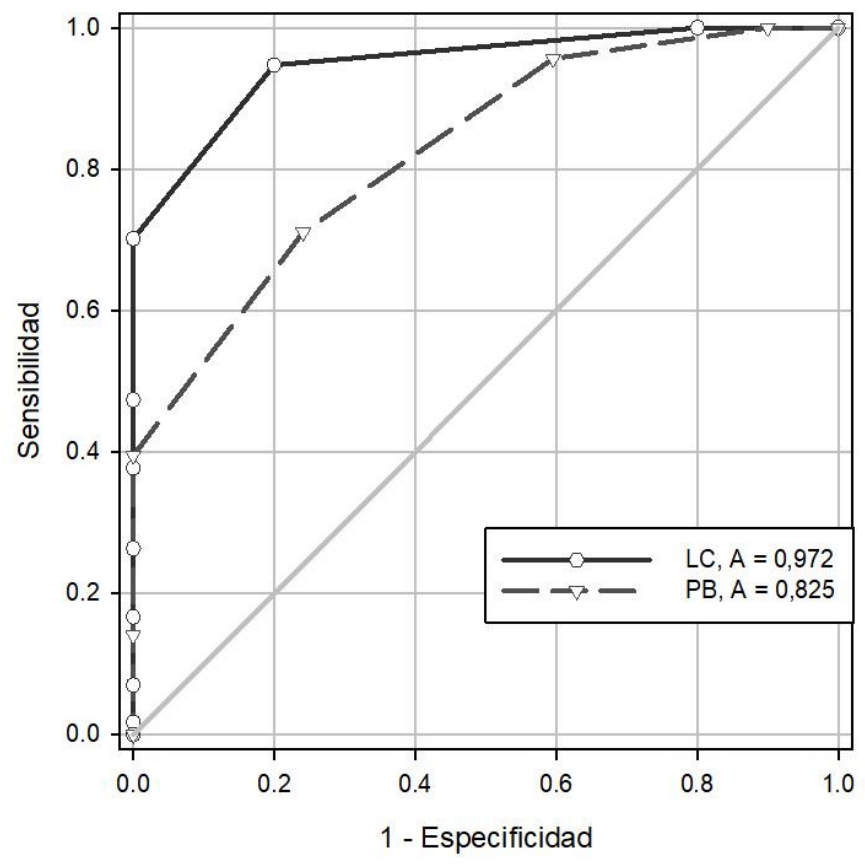

